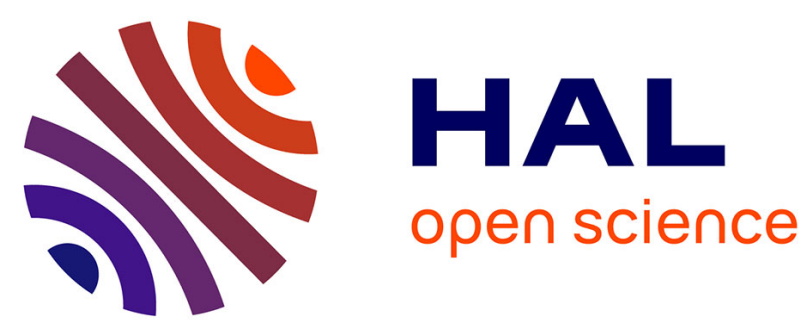

\title{
Assessment of Digital Image Correlation Measurement Accuracy in the Ultimate Error Regime: Improved Models of Systematic and Random Errors
}

\author{
Michel Bornert, Pascal Doumalin, Jean-Christophe Dupré, Christophe
} Poilâne, Laurent Robert, Evelyne Toussaint, Bertrand Wattrisse

\section{To cite this version:}

Michel Bornert, Pascal Doumalin, Jean-Christophe Dupré, Christophe Poilâne, Laurent Robert, et al.. Assessment of Digital Image Correlation Measurement Accuracy in the Ultimate Error Regime: Improved Models of Systematic and Random Errors. Experimental Mechanics, 2018, 58 (1), p.33-48. 10.1007/s11340-017-0328-5 . hal-01577239

\section{HAL Id: hal-01577239 \\ https://hal.science/hal-01577239}

Submitted on 25 Aug 2017

HAL is a multi-disciplinary open access archive for the deposit and dissemination of scientific research documents, whether they are published or not. The documents may come from teaching and research institutions in France or abroad, or from public or private research centers.
L'archive ouverte pluridisciplinaire HAL, est destinée au dépôt et à la diffusion de documents scientifiques de niveau recherche, publiés ou non, émanant des établissements d'enseignement et de recherche français ou étrangers, des laboratoires publics ou privés. 


\title{
Assessment of Digital Image Correlation Measurement Accuracy in the Ultimate Error Regime: Improved Models of Systematic and Random Errors
}

\author{
M. Bornert ${ }^{1} \cdot$ P. Doumalin ${ }^{2} \cdot$ J.-C. Dupré ${ }^{2} \cdot$ C. Poilâne ${ }^{3} \cdot$ L. Robert ${ }^{4} \cdot$ E. Toussaint ${ }^{5} \cdot$ B. Wattrisse ${ }^{6}$
}

\begin{abstract}
The literature contains many studies on assessment of DIC uncertainties, particularly in the ultimate error regime, when the shape function used to describe the material transformation perfectly matches the actual transformation. For pure sub-pixel translations, bias and random errors obtained for experimental or synthetic images show more complex evolution versus the fractional part of displacement than those predicted by the existing theoretical models. Indeed, small deviations arise, mainly around integer values of imposed displacements for noisy images, and they are interpreted as the unrepresentativeness of the underlying hypotheses of the theoretical models. In a first step, differences between imposed
\end{abstract}

On behalf of the "Metrology" Workgroup of the French CNRS research network 2519 "Mesures de Champs et Identification en Mécanique des Solides / Full-field measurements and identification in solid mechanics".

This paper is dedicated to the memory of our colleague Laurent Robert who animated the "Metrology" Workgroup of the GdR2519. He passed away on April 15th, 2015 after a long fight against myeloma cancer.

P. Doumalin

pascal.doumalin@univ-poitiers.fr

1 Laboratoire Navier, UMR 8205, CNRS, ENPC, IFSTTAR, Université Paris-Est, Marne-la-Vallée, France

2 Institut P', UPR 3346 CNRS, Université de Poitiers, SP2MI, Futuroscope Chasseneuil, France

3 CIMAP, UMR 6252, CNRS, CEA, Université de Caen Basse-Normandie, ENSICAEN, Caen, France

4 Université de Toulouse; Mines Albi, INSA, UPS, ISAE; ICA (Institut Clément Ader), Campus Jarlard, F-81013 Albi Cedex 09, France

5 Institut Pascal, UMR6602, CNRS, Université Blaise Pascal - IFMA, Aubière, France

6 Laboratoire de Mécanique et Génie Civil, UMR CNRS 5508, Université Montpellier 2, Montpellier, France and measured displacements are analysed: random error is independent of fractional displacement, and systematic error does not decrease for values close to integer displacements whatever the noise level. Therefore, new prediction models are proposed based on the analysis of identified phenomena from synthetic speckle-pattern 8-bit images. The statistical approach used in this paper generalizes the methods proposed in the literature and mimics the experimental methodology usually used for displacement measurements performed in different subsets in the same image. Two closed-form expressions for the systematic and random errors and a linear interpolation scheme are developed. These models, depending only on image properties and the imposed displacement, are built with a very limited number of parameters. It is then possible to predict the evolution of bias and random errors from one to four images.

Keywords Digital image correlation · Uncertainty quantification · Prediction models · Error assessment . Ultimate error regime

\section{Introduction}

Full-field kinematic measurements by digital image correlation (DIC) have become an unavoidable technique in experimental solid mechanics for characterizing the local strain mechanisms in materials and structures. Initially developed for planar surfaces [1-3], these methods have been extended to tackle curved surfaces using stereovision algorithms $[1,4$, 5]. More recently, kinematic volume measurements have become available in academic and industrial research centres due to the democratization of different tomographic devices (optical and X-ray tomography [6-9], confocal microscopy [10], magnetic resonance imaging [11],...). Other developments 
concern improving correlation algorithms by regularizing the displacement fields using diverse strategies: global approaches ensuring displacement continuity $[12,13]$ and integrated approaches additionally enforcing the local equilibrium associated with a given behaviour law [14].

As usual in experimental physics, the measurement of one datum must be related to its uncertainty. In the context of DIC, the assessment of displacement measurement uncertainties is delicate due to the complexity of the "measurement" chain, which involves: (i) the imaging device and the image itself and (ii) the models used in processing the entire image (camera model, image similitude model, image transformation model, image interpolation model, image noise model...).

The characterization of metrological performances of DIC techniques has thus been widely studied from an experimental and theoretical point of view. Due to the complexity of the problem, these approaches generally address a limited number of parameters of the overall DIC problem. For instance, from an experimental point of view, the camera and speckle images are fixed and the study is limited to the effect of image interpolation and image noise [15-18]. The first theoretical approaches proposed a noise propagation analysis in the DIC procedure [19]. They were later complemented by adding the effect of image interpolation [20]. The collaborative work performed by the "Metrology" workgroup of the CNRS research network 2519 aims at contributing to a systematic approach to this question by dissociating the steps of the measurement chain. In [21], the effect of the image transformation was investigated. Two error regimes can be encountered: (i) the ultimate error regime, if the shape function is rich enough to represent the actual displacement field, and (ii) the shape function mismatch regime, if the shape function is too poor to reproduce the real displacement field on the chosen subset. A new study [22] examined the influence of several options in the DIC process (criterion, direct or inverse computation, optimization algorithm) on metrological performance using an error propagation strategy similar to the one proposed in [20]. In this approach, the shape of the correlation criterion is not investigated and errors due to the possible presence of local minima are not considered. The study in [23] completes the previous works by highlighting differences between spatial and temporal random errors and by introducing the convex character of their evolution versus the fractional part of the imposed displacement. Nevertheless, this approach cannot explain the values of random error close to integer values of the imposed displacement as shown in [21]. In this paper, we thoroughly investigate the ultimate error regime and we develop simple models that consider the possible non-convexity of correlation criterion to describe the evolution of systematic and random errors with respect to image gradient, image noise and imposed displacement. Our approach generalizes the methods proposed by Wang et al. in [20] to better reproduce the experimental methodology classically used to obtain systematic and random errors.

\section{Existing Models for DIC Ultimate Error}

\section{Error Analysis Based on Continuous Image Models}

Many experimental characterizations of the ultimate error regime can be found in the literature for pure rigid body translations [16-18, 24-38]. Conversely, very few theoretical approaches have been proposed. A first analysis was proposed by Roux and Hild [19]. It consists of a statistical description of the sensitivity of displacement measurements with respect to superimposed image noise. This approach required two major hypotheses: a perfect reconstruction of the grey level images, and a statistical model of the image noise (a zero-mean with a $\sigma_{n}$ standard deviation for uniform white noise). The perfect reconstruction of the initial image $I$ and the transformed one $T$ corresponds to the ideal situation in which both $I(X)$ and $T(X)$ are known whatever the position $X$. Moreover, the optical flow is perfectly conserved, i.e., $I(X)=T(X+u(X))$, where $u(X)$ represents the actual transformation of the subset near $X$. The authors suggested an image noise propagation in an analytical description of the correlation criterion (namely, the usual "sum of squared difference" SSD criterion). They found that the average of the displacement error $\left(\overline{\Delta u}^{c}\right)$ was equal to zero and that the standard deviation of the displacement error $\left(\sigma_{u}^{c}\right)$ was proportional to the standard deviation of the image noise $\left(\sigma_{n}\right)$ and inversely proportional to the average of the squared grey level gradients $\left(\overline{\nabla I^{2}}\right)$ and to the subset size $d$ (see equation (1)):

$\left\{\begin{array}{l}\overline{\Delta u}^{c}=\sigma_{n} \\ \sigma_{u}^{c} \alpha \frac{\sigma_{n}}{d \sqrt{\overline{\nabla I^{2}}}}\end{array}\right.$

Note that the $c$ exponent stands for "continuous image model".

\section{Error Analysis for Images with Finite-Sized Pixels}

More recently, Wang et al. [20] have extended this approach to more realistic situations by introducing grey level interpolation, required in most DIC software, in the analytical expression of the displacement error. They considered a uniform white noise (zero-mean, $\sigma_{n}$ standard deviation), and two types of classical grey level interpolations, namely, linear and cubic polynomial interpolation. Higher order interpolations have not been considered 
because they lead to overly complex analytical expressions. For each interpolation, they propagated the image noise in the correlation formulation (also based on the SSD criterion), to obtain the closed form of the displacement error for a given subset (i.e., with a fixed spatial distribution of grey levels uncorrupted by the superimposed random noise). They defined the systematic error as the expectation of the displacement error over all noise realizations, and the random error as the variance of the displacement error. By considering a perfect reconstruction of the grey level images, the authors obtained the same expressions for the systematic and random errors as in [19]. If an imperfect reconstruction based on interpolation of grey levels was considered, the expression of random errors was also the same, and in that case the systematic error was not null:

$$
\left\{\begin{array}{l}
\sigma_{u}^{d}{ }^{\alpha} \frac{\sigma_{n}}{d \sqrt{\overline{\nabla I^{2}}}} \\
\overline{\Delta u}^{d}=-\frac{\overline{h(\tau) \nabla I}}{\overline{\nabla I}^{2}}+f_{i}(\tau) \frac{\sigma_{n}^{2}}{\overline{\nabla I}^{2}}
\end{array}\right.
$$

Here, the $d$ exponent stands for "discontinuous (interpolated) image model".

For this model, the random error $\sigma_{u}^{d}$ is also independent of the actual displacement. The first part of the expression for the systematic error $\overline{\Delta u}^{d}$ is noiseindependent and shows a dependence on the fractional part $\tau$ of the imposed displacement through the local grey level residual $h(\tau)$ at the correlation optimum. The second part shows a quadratic dependence on noise. The function $f_{i}$ depends on the chosen grey level interpolation: it is linear for a linear interpolation and quintic for a cubic one. Note that in this expression $\bar{A}$ stands for the average of $A$ for a set of drawings over the given subset. Of note is that this way of defining the systematic error differs from the classical experimental or numerical approaches in which the systematic error is averaged over all the subset realizations, with various grey level and random noise distributions.

\section{Enriched Modelling of Random Error Analysis}

\section{Characterization of Random Error}

To be consistent with the theoretical analyses discussed in the previous section, a basic subset-based DIC code has been implemented. It involves an SSD criterion along with a bilinear image interpolation (bi-cubic interpolations have also been implemented and will be used in section 3.3). As this paper focuses only on the ultimate error regime, only pure sub-pixel translations of images were investigated with constant shape functions. A first-order gradient-based algorithm was used to minimize the criterion for each subset.

Regarding statistical analysis, the methodology is identical to the one proposed in [21]: the same synthetic speckle-pattern 8-bit images were used. Speckle patterns of three mean radii $r$ were generated $\left(r=r_{0} / 2, r=r_{0}\right.$ and $r=2 r_{0}$ with $r_{0} \approx 2.2 \mathrm{px}$ ) and the size of the images with respect to the speckle size was kept constant (e.g., $512 \times 512 \mathrm{px}$ definition for the $r_{0} / 2$ speckle size and so on, as in [21, 36, 39]). A uniform Gaussian white noise with five intensity levels (standard deviation $\sigma_{n}=0,2,4$, $8,16 \mathrm{GL})$ has been added to the pixel grey levels. The statistical analysis was based on the computed standard deviation $\sigma_{u}$ (random error) and the arithmetic mean $\overline{\Delta u}$ (systematic error, or bias) of the displacement error at the centre of a correlation window of coordinates $(i, j)$ defined by:

$\Delta u_{i j}=u_{i, j}^{\text {measured }}-u_{i, j}^{\text {imposed }}$

where $u_{i, j}^{\text {measured }}$ is the evaluation of the displacement field provided at this position by the DIC package. The correlation is performed on non-overlapping subsets to ensure the statistical independence of measurements. The standard deviation $\sigma_{u}$ is calculated by:

$\sigma_{u}=\sqrt{\frac{n \sum_{i, j} \Delta u_{i j}^{2}-\left[\sum_{i, j} \Delta u_{i j}\right]^{2}}{n(n-1)}}$

where $n$ is the number of positions $(i, j)$ for which the displacement is evaluated, whereas the arithmetic mean is obtained as:

$\overline{\Delta u}=\frac{\sum_{i, j} \Delta u_{i j}}{n}$

Figure 1 presents the evolution of the random error function of the prescribed displacement $\tau$ for various image noises, obtained for the standard speckle size $\left(r=r_{0}\right)$ and for the reference subset size $d=16 \mathrm{px}$.

Three main observations can be highlighted:

(i) as previously reported ([39] and references within), the amplitude of the random error increases systematically with the amplitude of the image noise;

(ii) the amplitude of the random error depends on the prescribed sub-pixel displacement $\tau$,

(iii) the shape of the random error curve evolves with the noise level: for noiseless images or small 


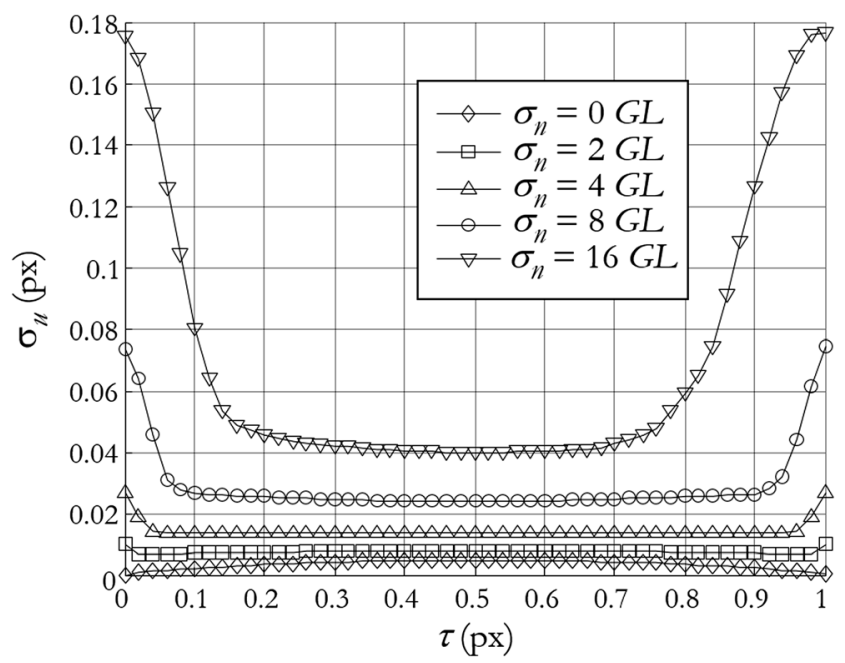

Fig. 1 Evolution of the random error function of the prescribed displacement $\tau$ for the five image noises $\sigma_{n}=0,2,4,8,16 \mathrm{GL}$, obtained for the standard speckle size $\left(r=r_{0}\right)$ and for the reference subset size of $d=16$ pix

image noise, a typical bell-shape (concave) curve is observed with almost zero error for integer displacement $(0$ or $1 \mathrm{px})$ and a maximum error for a displacement of $0.5 \mathrm{px}$. For noisy or very noisy images a U-shape (convex) curve is observed with maximum errors for integer displacement values. For each level of noise, the two shapes show a relatively constant plateau centred on $\tau=0.5 \mathrm{px}$. The size of this plateau can be expressed using the parameter $\delta_{X}^{m}$ (superscript $m$ stands for "measured") such that $\sigma_{u}$ remains approximately constant in the interval $\left[\delta_{X}^{m} ; 1-\delta_{X}^{m}\right]$. In Fig. $1, \delta_{X}^{m}$ is approximately equal to $0.2 \mathrm{px}$ for $\sigma_{n}=16 \mathrm{GL}$.

Importantly, in contrast to items (ii) and (iii), item (i) is consistent with the random error predictions of equations (1) and (2). Here we will demonstrate the consistency of the results in the plateau with the predictions given by equations (1) and (2). We will then focus on the behaviour of the DIC algorithm near integer displacement values. Finally, the third sub-section will be devoted to the proposition of a phenomenological model to describe all the dependencies (noise and imposed displacement) of the random error.

\section{Comparison to Existing Models Close to $\tau=0.5$ px}

According to the theoretical predictions associated with equations (1) and (2), the random error is only a function of the average of the squared grey level gradients $\overline{\nabla I^{2}}$, the noise level $\sigma_{n}$ and the correlation subset size $d$. By calculating $\overline{\nabla I^{2}}$ on noiseless images for the three investigated speckle sizes $\left(r_{0}\right)$
$2, r_{0}$ and $2 r_{0}$ ), we obtain the numerical values given in Table 1 . Results presented in this table show that the term $d \sqrt{\overline{\nabla I^{2}}}$ is quasi-constant, whatever the speckle size.

A consequence of this observation is that the measured random error $\sigma_{u}$ should vary linearly with respect to the image noise $\sigma_{n}$, according to equations (1) and (2). The slope should be constant whatever the speckle size. This analysis is confirmed by the results presented in Fig. 2, which presents the evolution of the random error $\sigma_{u}^{0.5}$ computed for the imposed displacement $\tau=0.5 \mathrm{px}$ with respect to the actual image noise equal to $\frac{1}{6}+\sigma_{n}^{2}$ (see reference [38]). This definition considers the noise induced by the quantification of the image. The slopes of the straight lines ( $\alpha$ in Fig. 2 ) are also given in Table 1.

Results presented in Table 1 and Fig. 2 highlight the consistency of the theoretical model proposed in equations (1) and (2) with regard to the numerical estimation of the random error $\sigma_{u}$ estimated for $\mathrm{ml} \leq \tau \leq 1-\mathrm{ml}$ $\delta_{X}^{m} \leq \tau \leq 1-\delta_{X}^{m}$ px. However, this consistency fails for the smallest speckle size $\left(r=r_{0} / 2\right)$ due to the sub-sampling of the texture signal that induces a corrupted estimation of the image gradients. Indeed, the Y-intercept of the curve corresponding to $r=r_{0} / 2$ is significantly higher than that of classical speckle sizes (e.g., for $r=r_{0}$ or $r=2 r_{0}$ ). The theoretical model predicts a null random error for noiseless images whatever the speckle size.

Similarly, the difference between the model and numerical results slightly increases for high noise levels, probably because the noise is then no longer averaged on the chosen subsets. Consequently, the expectancy of the random variable $\sigma_{u}$ computed from a limited set of draws in the image (approximately 4000) is not correctly estimated for high noise level, contrary to the case for low noise level.

In conclusion, equations (1) and (2) are valid only when the imposed displacement $\tau$ is sufficiently different from integer pixel values (roughly $\tau$ comprised between $\delta_{X}^{m}$ and $1-\delta_{X}^{m}$ ). Subsequent sub-sections present an extension of the theoretical model relative to the random error for imposed displacements close to 0 and $1 \mathrm{px}$ (namely, $0 \leq \tau \leq \delta_{X}^{m}$ and $1-\delta_{X}^{m} \leq \tau \leq 1$ ).

Table 1 Statistical description of the speckle images and slope of the lines $\alpha$ in Fig. 2

\begin{tabular}{lllll}
\hline$d(\mathrm{px})$ & $r$ & $\overline{\nabla I^{2}}(\mathrm{GL} / \mathrm{px})^{2}$ & $\left(d \sqrt{\overline{\nabla I^{2}}}\right)\left[\mathrm{GL}^{-1}\right]^{-1}$ & $\begin{array}{l}\text { Slopes of } \\
\text { lines in } \\
\text { Fig. 2 } \\
{[p x / G L]}\end{array}$ \\
\hline 8 & $r_{0} / 2$ & 3000 & $2.3 \mathrm{E}-3$ & $2.55 \mathrm{E}-3$ \\
16 & $r_{0}$ & 90 & $2.1 \mathrm{E}-3$ & $2.29 \mathrm{E}-3$ \\
32 & $2 r_{0}$ & 250 & $2.0 \mathrm{E}-3$ & $1.69 \mathrm{E}-3$ \\
\hline
\end{tabular}




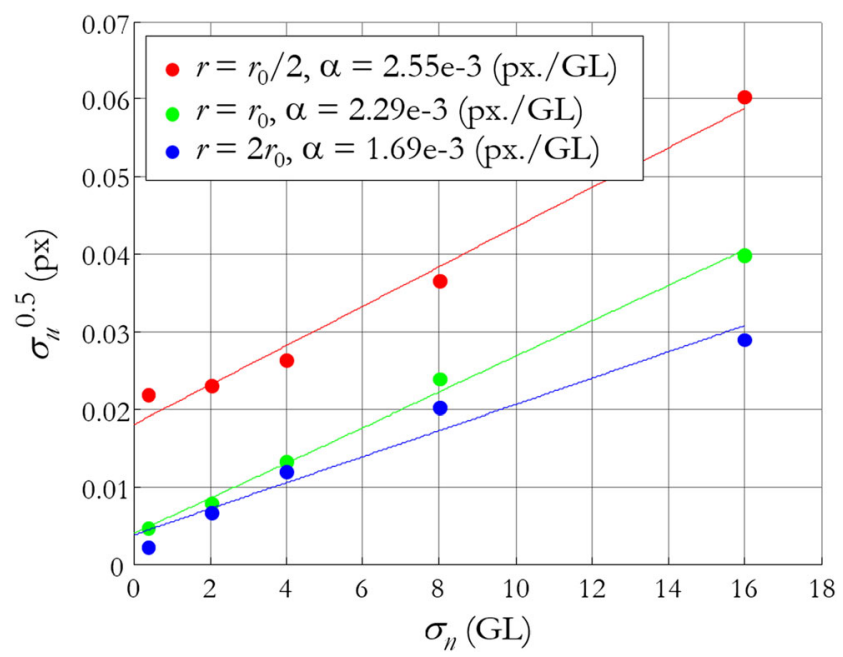

Fig. 2 Experimental random error $\sigma_{u}^{0.5}=\sigma_{u}(\tau=0.5 \mathrm{px})$ versus noise level $\sigma_{n}$ for various speckle sizes and associated subset sizes

\section{Analytical Modelling of Observed Behaviour for SSD Criterion at $\tau=\mathbf{0}$ pix}

To explain higher values of $\sigma_{u}$ for imposed integer displacements, the situation $\tau=0 \mathrm{px}$ is studied. In this case, the conservation of optical flow induces $T\left(X_{i}\right)=I\left(X_{i}\right)$. In the following, the analysis is restricted to a linear interpolation scheme, so that $T\left(X_{i}+\delta_{X}\right)=$ $T\left(X_{i}\right)+\delta_{X}\left[T\left(X_{i+1}\right)-T\left(X_{i}\right)\right]=T\left(X_{i}\right)+\delta_{X} \nabla T\left(X_{i}\right)$. In the presence of image noises $\varepsilon_{1}\left(X_{i}\right)$ and $\varepsilon_{2}\left(X_{i}\right)$ on the initial and final images, respectively, the intensities become $\tilde{I}\left(X_{i}\right)$ $=I\left(X_{i}\right)+\varepsilon_{1}\left(X_{i}\right)$ and $\tilde{T}\left(X_{i}\right)=T\left(X_{i}\right)+\varepsilon_{2}\left(X_{i}\right)$. It is then possible to determine the analytical expression of the SSD correlation coefficient with $\delta_{X}$ and then to minimize this expression. The SSD criterion writes as:

$\operatorname{SSD}\left(\delta_{X}\right)=\sum_{i}\left(I\left(X_{i}\right)-T\left(X_{i}+\delta_{X}\right)\right)^{2}$

For the noiseless case, the criterion becomes:

$\operatorname{SSD}\left(\delta_{X}\right)=N \overline{\nabla T^{2}} \delta_{X}^{2}$

where $N$ is the number of pixels in the correlation subset $d$ and $\overline{\nabla T^{2}}$ is the average of the square value of the grey level gradients in $d$ in the final image. The latter is supposed to be constant over the entire image. The criterion is a (convex) parabola that is minimal for $\delta_{X}=0$. With a superimposed image noise, the criterion becomes:

$\operatorname{SSD}\left(\delta_{X}\right)=\sum_{i}\left\{I\left(X_{i}\right)+\varepsilon_{1}\left(X_{i}\right)-\left(T\left(X_{i}\right)+\varepsilon_{2}\left(X_{i}\right)+\delta_{X}\left[\nabla T\left(X_{i}\right)+\nabla \varepsilon_{2}\left(X_{i}\right)\right]\right)\right\}^{2}$

Provided that (i) image noises $\varepsilon_{1}\left(X_{i}\right)$ and $\varepsilon_{2}\left(X_{i}\right)$, and image grey levels $I\left(X_{i}\right)$ and $T\left(X_{i}\right)$ are independent random variables, (ii) the image noise is zero-mean and (iii) the correlation subset is large enough to get a good estimate of the expectancy of the random variables, equation (8) is written as:

$\operatorname{SSD}\left(\delta_{X}\right)=2 N \sigma_{n}^{2}-2 N \sigma_{n}^{2} \delta_{X}+N\left(2 \sigma_{n}^{2}+\overline{\nabla T^{2}}\right) \delta_{X}^{2}$

Figure 3 presents the evolution of the correlation criterion, directly evaluated from our DIC software, with the transformation parameter $\delta_{X}$ for the noiseless case (Fig. 3(a)) and for a noisy one (Fig. 3(b), $\sigma_{n}=16 \mathrm{GL}$ ), as computed in equation (6) for bi-linear and bi-cubic polynomial interpolations. In this figure, the evolution of the correlation criterion is also plotted for bi-cubic polynomial interpolation to demonstrate that nonconvexity is always observed for high-order interpolation schemes.

The case presented in Fig. 3 corresponds to images for which $r=r_{0}$. Initial and final image grey levels are statistically equivalent so that $\overline{\nabla T^{2}} \approx \overline{\nabla I^{2}}=900(\mathrm{GL} / \mathrm{px})$ 2. The evolution of the correlation criterion $\operatorname{SSD}\left(\delta_{X}\right)$ is computed for the subset located in the centre of the image and a subset size $d=256$ px (Fig. 3(a, b)). Thus, the influence of noise on the minimum of the correlation coefficient is investigated for a fixed image pattern. For such a subset size, the assumption that the image noise is averaged on the subset is valid and equation (9) holds true. Figure 3(a, b) clearly demonstrate the parity of the correlation coefficient in both cases, and the apparition of two local minima for the noisy case. The presence of image noise modifies the shape of the criterion that is no longer convex. It also confirms that the correlation coefficient is quadratic on 1pixel wide segments for bilinear interpolation. Equation (9) is only valid for $\delta_{X} \in[0,1]$, and the theoretical position of the minimum given by the analytical minimization of equation (9), $\delta_{X}^{\text {th }}$, is written as:

$\delta_{X}^{t h}=\sigma_{n}^{2} /\left(2 \sigma_{n}^{2}+\overline{\nabla T^{2}}\right)$

In the present case, $\delta_{X}^{\text {th }} \approx 0.181 \mathrm{px}$. This value is consistent with the value $\delta_{X}^{m}$ of the minimum of SSD criterion, at $\delta_{X}^{m} \approx 0$. 180 in Fig. 3(b). Using smaller subsets, a deviation with respect to equation (10) appears because of the particular realization of the noise on the subset and because the correlation subset is not large enough to ensure a good estimate of the noise expectation. This effect is observed in Fig. 3(c), which represents the correlation coefficient normalized by the subset area for two subset sizes ( $d=16$ and $256 \mathrm{px})$ and for the two polynomial interpolations. This normalization allows comparing SSD criteria whatever the subset size. The deviation with respect to theoretical results can be clearly observed from the loss of symmetry of the correlation criteria. For $d=16 \mathrm{px}$ and using the bilinear interpolation, we obtained $\delta_{X}^{m} \approx 0.193 \mathrm{px}$ for the positive minimum and $\delta_{X}^{m} \approx-0.167 \mathrm{px}$ for the negative one. 
(a)

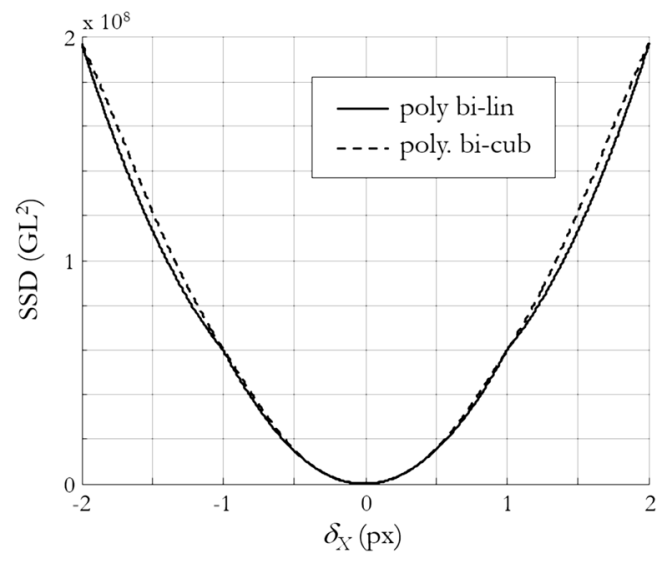

(b)

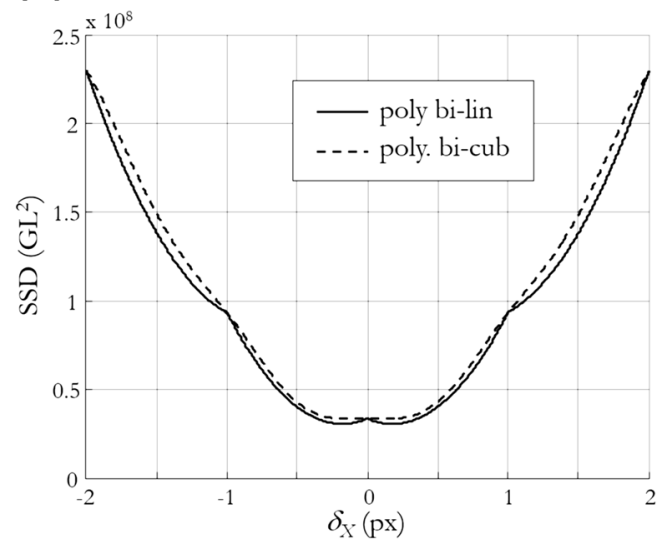

(c)

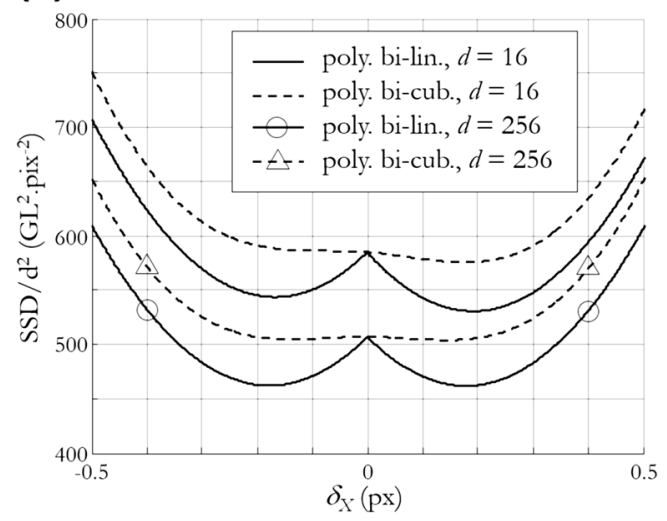

Fig. 3 Evolution of the SSD correlation coefficient with $\delta_{X}$ for $\tau=0$ and for bi-linear and bi-cubic polynomial interpolations for one subset: (a) $\mathrm{d}=256 \mathrm{px}$ without noise; (b) $\mathrm{d}=256 \mathrm{px}$ with noise $\sigma_{n}=16 \mathrm{GL} ;(\mathbf{c})$ evolution with $\delta_{X}$ of the SSD correlation coefficient normalized by $\mathrm{d}^{2}$ for two subset sizes $(\mathrm{d}=16 \mathrm{px}$ and $\mathrm{d}=256 \mathrm{px})$ with noise, $\sigma_{n}=16 \mathrm{GL}$

For sufficiently large subsets, the correlation coefficient is even and the values $\delta_{X}^{m} \approx 0.181 \mathrm{px}$ and $\delta_{X}^{m} \approx-0.181 \mathrm{px}$ are equiprobable. In this case, the standard deviation of the measured displacement is equal to $0.181 \mathrm{px}$. To conclude, for noisy images and for a null displacement, the random error results from two contributions: the first one, governed by equation (1), is related to the propagation of the image noise and the second one stemmed from the loss of convexity of the criterion.

Of note is that the present approach can be applied to other correlation coefficients such as NSSD, CC, NCC (see reference [4]). For a null displacement and for a bilinear interpolation scheme, calculations become more complex, but they still lead to closed-form expressions of $\delta_{X}^{\text {th }}$ with respect to the different image characteristics $\left(\overline{\nabla T^{2}}, \sigma_{n} \ldots\right)$. Using these expressions for the same images and $\sigma_{n}=16 \mathrm{GL}$, we obtain $\delta_{X}^{\text {th }} \approx 0.177 \mathrm{px}$ for NSSD, $\delta_{X}^{\text {th }}=0 \mathrm{px}$ for $\mathrm{CC}$ and $\delta_{X}^{\text {th }} \approx 0.185 \mathrm{px}$ for NCC. Except for the $\mathrm{CC}$ criterion, each criterion gives a similar result. The CC criterion is piecewise linear, consequently the systematic errors for integer displacement are null, and the random error is null whatever the imposed displacement. As shown in Fig. 3(c), a high-order interpolation scheme leads to a smoother criterion shape but does not prevent its non-convexity.

\section{Phenomenological Extension of the Model for Arbitrary $\tau$}

In a situation with a high noise level (16 GL), Fig. 4 presents histograms of 3844 DIC-calculated results (subset size $d=16 \mathrm{px}$ ) for four imposed displacements in the interval $0<\tau<\delta_{X}^{\text {th }}$. For an imposed displacement $\tau$ very close to 0 , the histograms show a bimodal population associated with the two separate solutions previously discussed (non-convex correlation criterion). The peak that is the furthest from the solution regularly diminishes when increasing the imposed displacement and becomes negligible for a value greater than approximately $0.2 \mathrm{px}$ in the illustrated example. Let us note that for a null imposed displacement, the two peaks are not symmetric due to the problem of statistic representativeness for small subsets with a high noise level as previously mentioned.

To quantify the evolution of these two populations, we focus on averages $m_{l}$ and $m_{r}$, and standard deviations $\sigma_{l}$ and $\sigma_{r}$ of the "left" and "right" populations, respectively. Figure 5 shows the evolution of these quantities $\left(\sigma_{l}\right.$ and $\left.\sigma_{r}\right)$ according to applied displacement $\tau$ and compares them to the standard deviation $\sigma_{u}$ of displacement measurement on the entire population. As already observed in Fig. $1, \sigma_{u}$ obtained by the DIC process diminishes with $\tau$ for $\tau$ smaller than $0.2 \mathrm{px}$, whereas $\sigma_{l}$ and $\sigma_{r}$ remain constant. One can notice that these latter quantities are equal to the value of $\sigma_{u}$ associated with the displacement $\tau=0.5$ px (see Figs. 1 and 5). The evolution of $\sigma_{u}$ is then the result of the progressive prevalence of the right population compared to the left one.

The following phenomenological model is restricted to an imposed displacement range: $0<\tau<\delta_{X}^{\text {th }}$. Let us consider 
Fig. 4 Histograms of the calculated displacements for 16 GL image noise for: (a) $\tau=0 \mathrm{px}$; (b) $\tau=0.06 \mathrm{px}$; (c) $\tau=0.12 \mathrm{px}$; and (d) $\tau=0.18 \mathrm{px}$ (a)

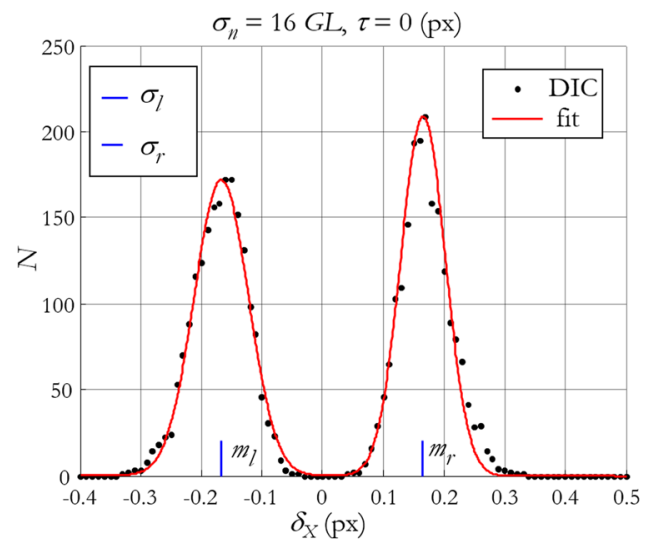

(c)

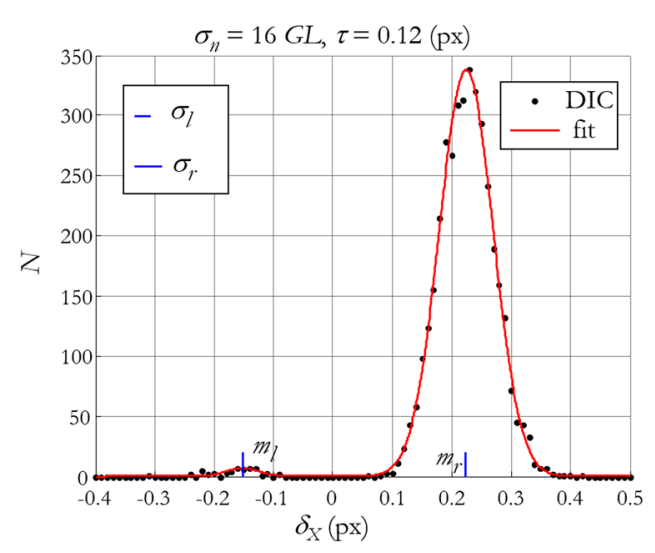

(b)

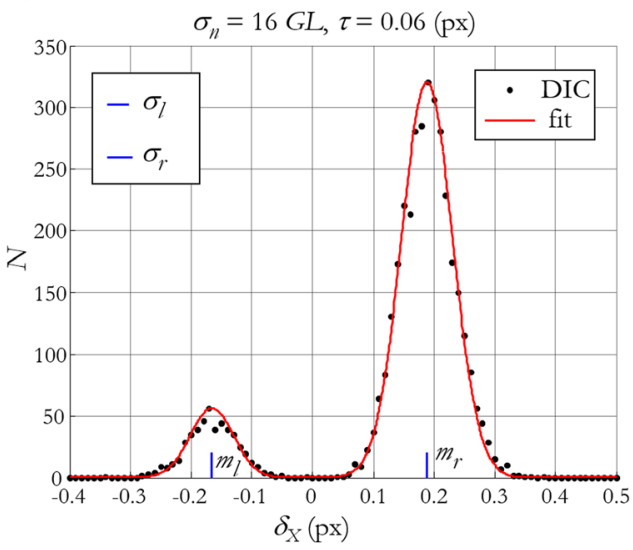

(d)

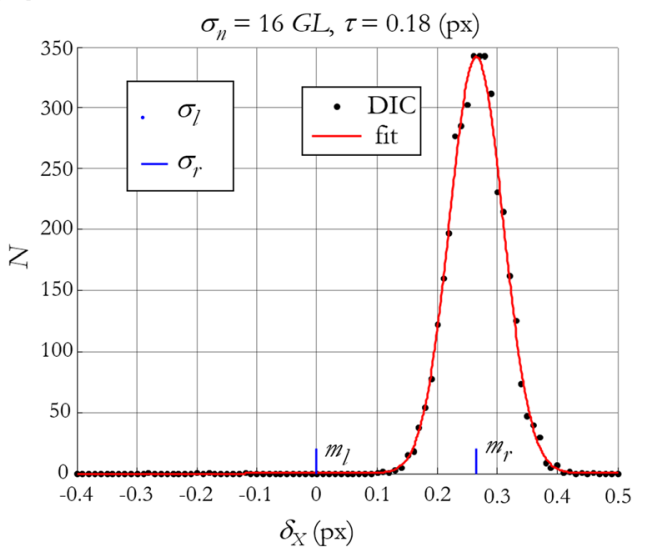

the subsequent assumptions: as shown in Fig. 4, in this interval, DIC measured values follow a bi-modal Gaussian distribution combining two density probability functions for the measured displacement $u$, denoted $p_{l}(u)$ and $p_{r}(u)$, associated with the prescribed displacement $\tau$. Let their expectations $(E[]$.$) and variances (V[]$.$) be: m_{l}(\tau)=E\left[p_{l}\right], m_{r}(\tau)=E\left[p_{r}\right]$ and $\sigma_{l}^{2}=V\left[p_{l}\right]=\sigma_{r}^{2}=V\left[p_{r}\right]$. From Figs. 4 and 5, we suppose that $\sigma_{l}$ and $\sigma_{r}$ do not depend on $\tau$ and are equal to $\sigma_{u}^{d}$. Figure 6 describes the evolution of the averages $m_{l}(\tau)$ and $m_{r}(\tau): m_{l}(\tau)$ stays constant for $\tau<\delta_{X}^{t h}$. The fact that $m_{l}(\tau)$ becomes null beyond $\delta_{X}^{\text {th }}$ is easily explained by the fact that the "left" population becomes very small (see Fig. 4(d)) and the fitting parameters of the Gaussian are set to zero in the absence of convergence. The evolution of $m_{r}(\tau)$ is quasi-linear. These evolutions can be modelled by equation (11):

$\left\{\begin{array}{c}m_{l}(\tau)=-\delta_{X}^{t h} \\ m_{r}(\tau)=\delta_{X}^{t h}+\tau\end{array}\right.$

We observed that the function $m_{r}(\tau)$ evolved quasi-linearly whatever the noise level. The slope of this evolution depends slightly on $\sigma_{n}$ : it evolves between 0.5 and 1 in the example tested here. In equation (11), the slope is set to 1 .

Both distributions in Fig. 4 are such that the total population $N_{0}$ is kept constant (i.e., constant number of correlated points whatever the imposed displacement). In this example, $N_{0}$ is equal to 3844 . We denote $N_{l}$ and $N_{r}$ the number of elements in the "left" and "right" populations, respectively:

$N_{l, r}=N_{0} \int_{-\infty}^{+\infty} p_{l, r}(t) d t$

As the total population is kept constant, the evolution of each population with respect to the imposed displacement variation can be written as:

$\left\{\begin{array}{c}N_{l}(\tau)=N_{0} / 2-\delta N(\tau) \\ N_{r}(\tau)=N_{0} / 2+\delta N(\tau)\end{array}\right.$

In equation (13), the quantity $\delta N$ stands for the variation in the number of points of populations $N_{l}$ and $N_{r}$. The numerical evolution of $\delta N$ as a function of $\tau$ is plotted with circles in Fig. 7(b). 


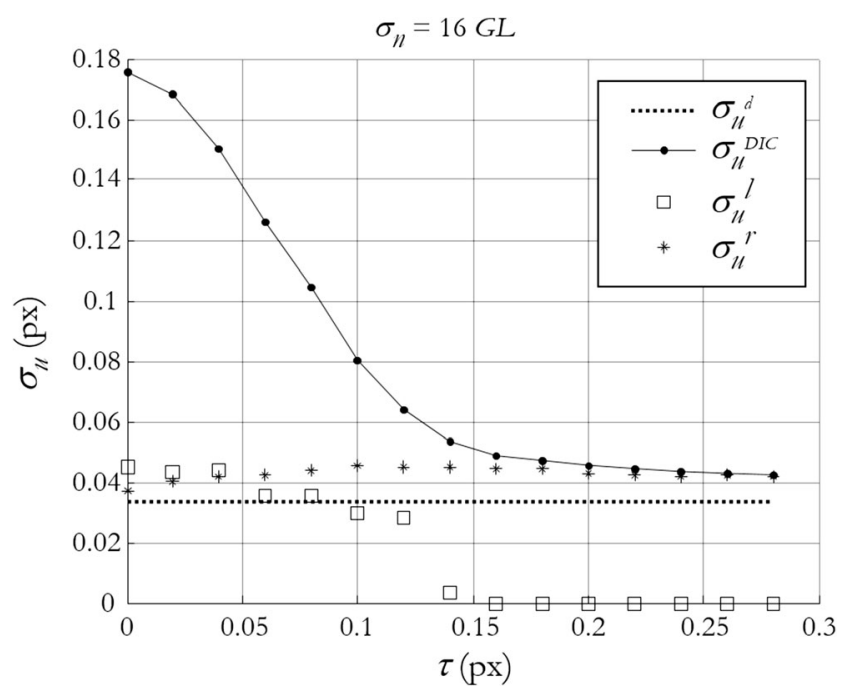

Fig. 5 Standard deviation of the "left" and "right" peaks and of the displacement measurement on the entire population versus imposed displacement $\tau$ for $\sigma_{n}=16 \mathrm{GL}$, which corresponds to $\sigma_{u}^{d}=0.033 \mathrm{px}$

As expected, $\delta N$ increases with $\tau$ from zero for a null $\tau$ and saturates for $\tau$ close to $\delta_{X}^{\text {th }}$. To be as generic as possible and to minimize the number of parameters introduced in the statistical description, we propose to parameterize the evolution of $\delta N$ using data coming only from the initial image and the noise level. The simplest model consists of assuming a linear evolution between points $(\tau=0 ; \delta N=0)$ and $\left(\tau=\delta_{X}^{\text {th }} ; \delta N=N_{0} / 2\right)$. Naturally, $\delta_{X}^{\text {th }}$ is given by equation (10), so one can write:

$\delta N(\tau)=N_{0} /\left(2 \delta_{X}^{t h}\right) \cdot \tau$

This basic (linear) model does not fit the numerical results shown in Fig. 7(b) (black solid line). For this set of images, an exponential model is more relevant. We propose:

$\delta N(\tau)=a\left(1-e^{-b \tau}\right)$

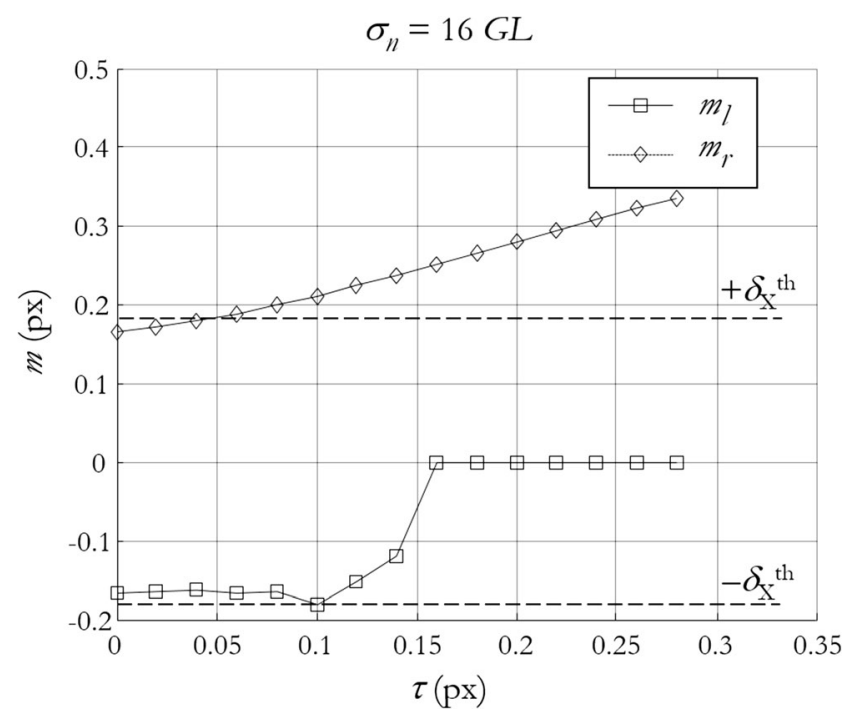

Fig. 6 Evolution of averages of both populations versus imposed displacement $\tau$

The fitted numerical values give $a=1978$ and $b=21.19 \mathrm{px}^{-1}$ (see solid line in Fig. 7(b)). To avoid the introduction of the set of new parameters $(a, b)$, one can also directly express the exponential function based only on the variables $N_{0}$ and $\delta_{X}^{\text {th }}$ that are already introduced in the model, such as $a=N_{0} / 2=1922$ and $b=4$ $/ \delta_{X}^{\text {th }}=22.1 \mathrm{px}^{-1}$ (see dashed line in Fig. 7(b)).

In the following, we define $u_{p}$ as the random variable representing the measured displacement. Consequently, the random error on the displacement corresponds to the standard deviation of $u_{p}: \sigma_{u_{p}}^{2}=V\left[u_{p}\right]=E\left[u_{p}^{2}\right]-E\left[u_{p}\right]^{2}$. Using the density probability function $p\left(u_{p}\right)=p_{l}\left(u_{p}\right)+p_{r}\left(u_{p}\right)$, we get:

$\sigma_{u_{p}}(\tau)=\frac{1}{N_{0}} \sqrt{\left\{N_{l}^{2}(\tau) \sigma_{l}^{2}+N_{r}^{2}(\tau) \sigma_{r}^{2}+N_{l}(\tau) N_{r}(\tau)\left[\left(m_{l}(\tau)-m_{r}(\tau)\right)^{2}+\sigma_{l}^{2}+\sigma_{r}^{2}\right]\right\}}$

Results of the random error of the displacement given by the proposed statistical models (linear or exponential case) of equations (11) to (16) superimposed with the measured values from the DIC computations are plotted in Fig. 8 as a function of the imposed displacement $\tau$ for $\sigma_{n}=16 G L$.

Figure 8 shows that the linear model is clearly unsatisfactory. Conversely, the exponential models are very consistent with the DIC data from simulated images. The exponential model is very satisfactory because it requires statistical information from only a single (e.g., reference) speckle image to compute the mean grey level gradients necessary to determine $\delta_{X}^{\text {th }}$ (see equation (10)) and $\sigma_{u}^{d}$ (see equation (2)). This model allows an explanation of the causes of the significant discrepancies observed between the classical model predictions [19, 20] near integer displacement values. It also allows extending these latter models to get a unified model giving a consistent expression for the random errors whatever the imposed displacement. In summary, the model of previous works [19] can be completed with the expression presented in this section. The random error can 
(a)

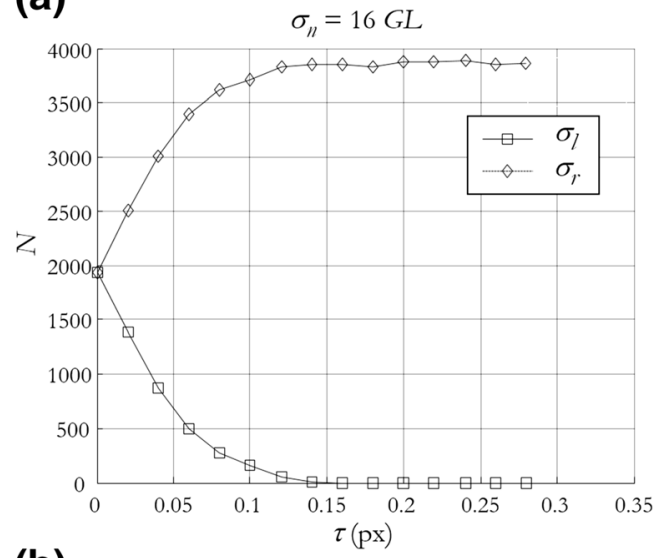

(b)

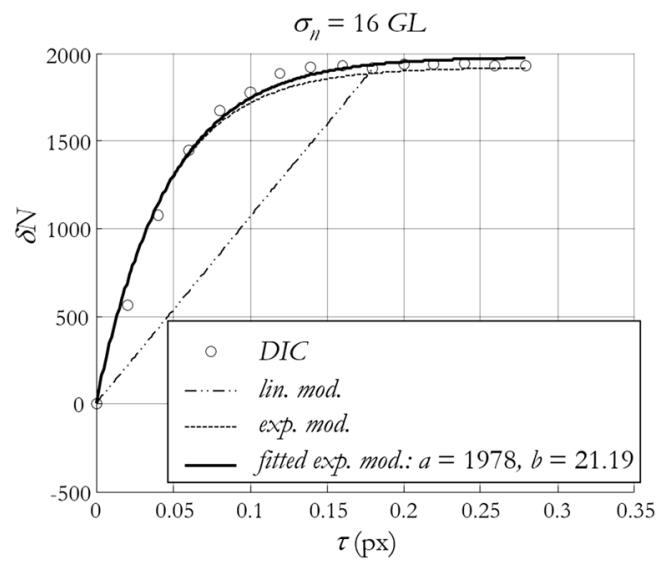

Fig. 7 (a) Evolution of the numbers of points of populations $N_{1}$ and $N_{r}$ versus imposed displacement $\tau$; and (b) modelling of the variation in the number of points of populations $\mathrm{N}_{\mathrm{l}}$ and $\mathrm{N}_{\mathrm{r}}$ versus imposed displacement $\tau$

be expressed as the maximum of the expression of $\sigma_{u}^{d}$ and $\sigma_{u_{p}}(\tau)$ given by equation (16):

$\sigma_{u}(\tau)=\max \left(\sigma_{u}^{d}, \sigma_{u_{p}}(\tau)\right)$

For a known image noise $\sigma_{n}$, the model proposed in equation (17) can be identified from at least two images. The initial image $(\tau=0)$ gives grey level gradients $\overline{\nabla T^{2}}$, and all parameters in equation (16), except $\delta N(\tau)$. When a fitted exponential model is used, at least two images are necessary to identify the two parameters $(a$ and $b)$ of the model. In this case, images with $\tau=\delta_{X}^{\text {th }} / 2$ and $\tau=\delta_{X}^{\text {th }}$ can be chosen. It is also possible to define the exponential model parameters $a$ and $b$ without any supplementary image with a known imposed displacement, using the expressions $a=N_{0} / 2$ and $b=4 / \delta_{X}^{\text {th }}$. The linear model can also be identified using a single image. The representativeness of this model can be improved by enriching the model expression and by increasing the number of images. In the latter case, the main difficulty is to impose a perfectly known displacement.

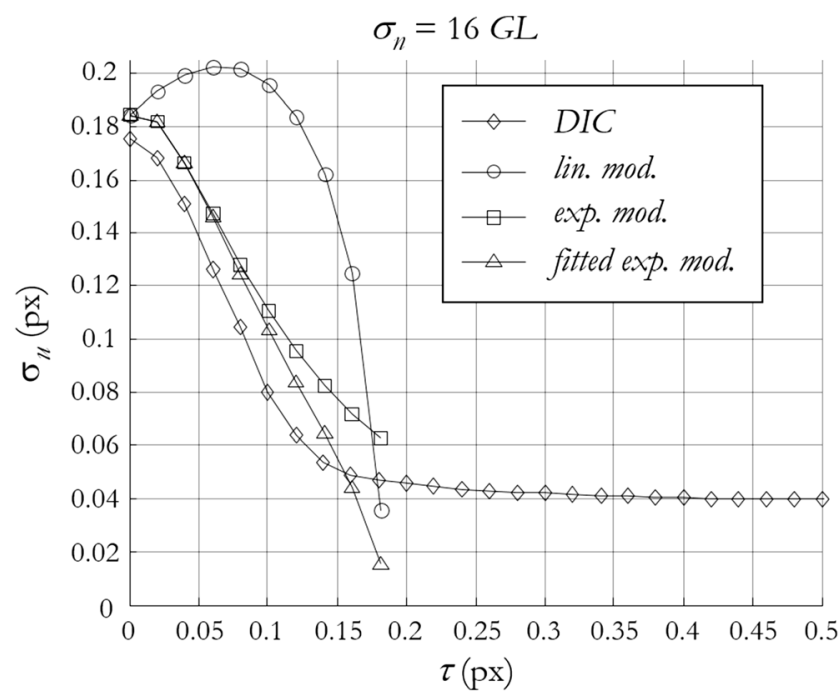

Fig. 8 Standard deviation of the displacement given by the proposed statistical models (linear or exponential) superimposed with the measured values from the DIC computations as a function of the imposed displacement $\tau$ for $\sigma_{n}=16 \mathrm{GL}$

\section{Enriched Modelling of Systematic Error Analysis}

\section{Reminder of Observed Behaviour: Consistency and Discrepancy with Existing Models}

Figure 9 presents the evolution of the systematic error as a function of the prescribed displacement $\tau$ for various image noises, obtained for the standard speckle size $\left(r=r_{0}\right)$ and for the reference subset size $d=16 \mathrm{px}$.

From this figure, three main observations can be highlighted:

(i) as previously reported ([38] and references within), the amplitude of the systematic error increases systematically with the amplitude of the image noise;

(ii) the amplitude of the systematic error depends on the prescribed sub-pixel displacement $\tau$ and classical Scurve shapes are observed, with a null error for $\tau=0$, 0.5 and $1 \mathrm{px}$ whatever the image noise;

(iii) the shape of the systematic error curve evolves with the noise level: for noiseless images or a small image noise, a typical S-curve similar to a sine curve is observed, with maxima and minima close to $\tau=0.2$ and $0.8 \mathrm{px}$. For noisy images, this sine-like shape evolves into an almost triangular-shaped curve as can be seen for image noise $\sigma_{n}=4,8$ and $16 \mathrm{GL}$. The most noticeable cases are provided for $\sigma_{n}=4$ and 8 GL with extrema sharply below 0.1 or above 0.9 px.

Importantly, in contrast to items (ii) and (iii), only item (i) is consistent with the systematic error predictions of equation (2). Indeed, equation (2) predicts a non-zero systematic error 


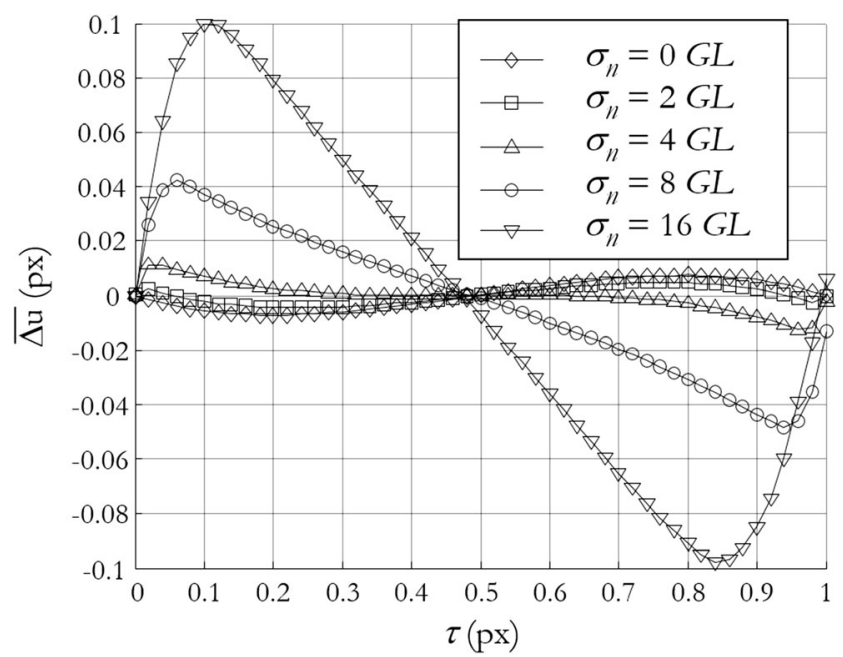

Fig. 9 Evolution of the systematic error versus the prescribed displacement $\tau$ for the five image noises $\sigma_{n}=0,2,4,8,16 \mathrm{GL}$, obtained for the standard speckle size $\left(r=r_{0}\right)$ and for the reference subset size of $d=16$ pix

for integer values of the imposed displacement $\tau$ in contrast to what is observed in Fig. 9.

In the following, we proposed to modify the analytical model of equation (2) given in [33] to make it more consistent with the observations from simulated images.

\section{A Modified Statistical Model for Systematic Error}

The same framework as the one introduced in [28] is used here, and we introduce: $I\left(X_{\mathrm{i}}\right)$, the grey level value in the initial image taken at integer pixel values $X_{\mathrm{i}}$, and $T\left(\beta_{\mathrm{i}}\right)$, the grey level value in the final transformed image taken at integer pixel values $\beta_{\mathrm{i}}$. DIC algorithms require non-integer pixel values to optimize the chosen correlation criterion. Various interpolation strategies are proposed in the literature: in the real space (linear or cubic polynomial interpolation; cubic, quintic or higher order splines, ...) or in the frequency space (using the translation properties of the Fourier transform, for example) [10-22, 24-26]. Under the abovementioned hypotheses, the interpolation error at pixel $X_{\mathrm{i}}$, denoted $e_{\mathrm{i}}(\tau)$, introduced by this operation is defined by:

$e_{i}(\tau)=I\left(X_{i}\right)-T\left(\beta_{i}+\tau\right)$

In the "ultimate error" regime, the errors are 1-pixel periodic so that the analysis can be restricted to a onepixel amplitude displacement interval. In the following, and as in subsection 3.3, we restrict the analysis to imposed displacements comprised of between 0 and 1 pixel so that one has $\beta_{i}=X_{i}$. Note that the interpolation error depends, a priori, on the applied displacement $\tau$.

The image grey levels are considered random variables characterized by their first and second statistical moments. It is supposed that the imposed displacement does not impact the image histograms, so that:

$$
\left\{\begin{array}{l}
\mathrm{E}\left[I\left(X_{i}\right)\right]=\mathrm{E}\left[T\left(X_{i}\right)\right]=\mathrm{E}\left[T\left(X_{i+1}\right)\right]=m_{G L} \\
\mathrm{~V}\left[I\left(X_{i}\right)\right]=\mathrm{V}\left[T\left(X_{i}\right)\right]=\mathrm{V}\left[T\left(X_{i+1}\right)\right]=\sigma_{G L}^{2}
\end{array}\right.
$$

These values depend only on the image characteristics. The additive intensity noise superimposed on the initial and final images, $\varepsilon_{1}\left(X_{\mathrm{i}}\right)$ and $\varepsilon_{2}\left(\beta_{\mathrm{i}}\right)$, is supposed to be uncorrelated white noise. The associated random variables (moments and covariance) verify:

$$
\left\{\begin{array}{l}
\mathrm{E}\left[\varepsilon_{1}\left(X_{i}\right)\right]=\mathrm{E}\left[\varepsilon_{2}\left(X_{i}\right)\right]=0 \\
\mathrm{~V}\left[\varepsilon_{1}\left(X_{i}\right)\right]=\mathrm{V}\left[\varepsilon_{2}\left(X_{i}\right)\right]=\sigma_{n}^{2} \\
\operatorname{cov}\left(\varepsilon_{1}\left(X_{i}\right), \varepsilon_{2}\left(X_{i}\right)\right)=0 \\
\operatorname{cov}\left(\varepsilon_{2}\left(X_{i}\right), \varepsilon_{2}\left(X_{i+1}\right)\right)=0
\end{array}\right.
$$

Furthermore, the image grey levels and their gradients are also supposed to be uncorrelated to the image noise.

Following the analysis developed in [33], the displacement error in DIC measurement $\Delta u=u-\tau$ can be written for a linear interpolation scheme as:

$$
\begin{aligned}
& \Delta u(\tau)=-\frac{\sum_{i}^{N}\left\{h\left(X_{i}\right) \nabla T\left(x_{i}\right)+h\left(X_{i}\right)\left[\varepsilon_{2}\left(X_{i+1}\right)-\varepsilon_{2}\left(X_{i}\right)\right]+\nabla T\left(x_{i}\right) \varepsilon_{2}\left(X_{i}\right)+\varepsilon_{2}\left(X_{i}\right) \varepsilon_{2}\left(X_{i+1}\right)\right\}}{\sum_{i}^{N} \nabla T^{2}\left(x_{i}\right)} \\
& -\frac{\sum_{i}^{N}\left\{\nabla T\left(X_{i}\right)\left[\varepsilon_{2}\left(X_{i+1}\right)-\varepsilon_{2}\left(X_{i}\right)\right] \tau-2 \varepsilon_{2}\left(X_{i+1}\right) \varepsilon_{2}\left(X_{i}\right) \tau-\nabla T\left(x_{i}\right) \varepsilon_{1}\left(X_{i}\right)-\varepsilon_{1}\left(X_{i}\right)\left[\varepsilon_{2}\left(X_{i+1}\right)-\varepsilon_{2}\left(X_{i}\right)\right]\right\}}{\sum_{i}^{N} \nabla T^{2}\left(x_{i}\right)} \\
& -\frac{\sum_{i}^{N}\left\{-\varepsilon_{2}^{2}\left(X_{i}\right)+\varepsilon_{2}^{2}\left(X_{i}\right) \tau+\varepsilon_{2}^{2}\left(X_{i+1}\right) \tau\right\}}{\sum_{i}^{N} \nabla T^{2}\left(x_{i}\right)}
\end{aligned}
$$

where:

- $\tau$ stands for the real imposed displacement between the initial and final images;
- $\quad u$ is the measured displacement between the initial and final images;

- $N$ is the number of pixels in the subset;

- $x_{i}=X_{i}+\tau$ 
- $\nabla T\left(x_{i}\right)=T\left(X_{i+1}\right)-T\left(X_{i}\right)$

- $h\left(X_{i}\right)=\left[T\left(X_{i}\right)+\nabla T\left(x_{i}\right) \tau\right]-I\left(X_{i}\right)$

The statistical analysis of equation (21) is the key to determining the systematic error associated with DIC: the systematic error is associated with the expectation of $\Delta u$. Wang et al. [20] considered a situation in which the statistical analysis was performed on several realizations of the image noise for a given grey level distribution (i.e., several image noise distributions for a fixed correlation subset). A more classical way of determining the systematic errors consists of considering the dual situation in which several realizations of the grey level distributions are considered for a given image noise distribution (i.e., several correlation subsets on two noisy initial and final - images). In this latter situation, the expectation should be calculated on all the realizations of the correlation subsets. We suppose that the correlation subset size is large enough to ensure that the quantity $\sum_{i}^{N} \nabla T^{2}\left(x_{i}\right)$ is constant whatever the subset position in the image, so that the denominator in equation (21) becomes:

$\sum_{i}^{N} \nabla T^{2}\left(x_{i}\right)=N \overline{\nabla T^{2}}$

where $\overline{\nabla T^{2}}$ represents the mean norm of the grey level gradient in the final image.

Recalling that the image grey levels $I$ and $T$ and their gradients are uncorrelated to the image noise 휀 1 and 휀 2 and that image noise between two images and between two adjacent pixels are also uncorrelated, the expectation of $\Delta u$ can be simplified from equation (21) as follows:

$\mathrm{E}[\Delta u(\tau)]=-\frac{\mathrm{E}\left[\sum_{i}^{N} h\left(X_{i}\right) \nabla T\left(x_{i}\right)\right]-N \sigma_{n}^{2}(1-2 \tau)}{N \overline{\nabla T^{2}}}$

Consequently, as $h\left(X_{i}\right)=-\left[I\left(X_{i}\right)-T\left(X_{i}+\tau\right)\right]=-e_{i}(\tau)$ for a linear interpolation and using the aforementioned assumptions on the statistical variables and variance properties, equation (23) becomes:

$\mathrm{E}[\Delta u(\tau)]=-\frac{1}{\overline{\nabla T^{2}}}[\underbrace{\operatorname{cov}\left(e_{i}(\tau), T\left(X_{i}\right)\right)-\operatorname{cov}\left(e_{i}(\tau), T\left(X_{i+1}\right)\right)}_{\mathrm{E}_{\text {interp }}}-\underbrace{\sigma_{n}^{2}(1-2 \tau)}_{\mathrm{E}_{\text {noise }}}]$

This equation clearly illustrates two contributions to the systematic error. The first one is associated with the interpolation bias (denoted $\mathrm{E}_{\text {interp }}$ ). This expression only depends on the noiseless image characteristics and it is expressed by the two covariance terms. The second one (denoted $\mathrm{E}_{\text {noise }}$ ) is related to the image characteristics and the image noise and varies linearly with $\tau$. In the following, the term $\mathrm{E}_{\text {interp }}$ will be referred to as the "interpolation bias error" and the term $\mathrm{E}_{\text {noise }}$ as the "noise bias error".

Equation (24) is different from the one proposed by Wang et al. in [20]. It is consistent with the classical procedure used to compute the S-shape systematic error curves (average error from a displacement field using a given subset size). Equation (24) includes displacement-independent parameters (initial image gradient, noise level) but also displacement-dependent quantities (the covariance terms). The estimation of these latter terms requires getting "precisely shifted" images.

\section{Comparison of the Proposed Model with DIC Data}

Figure 10 shows the evolution of the covariance terms in $\mathrm{E}_{\text {interp }}$ with the imposed displacement $\tau$. This result is directly provided by the calculus of each covariance term of equation (24) from the synthetic images used in this work with $r=r_{0}$. It clearly demonstrates that these covariances are not equal and that their difference evolves with $\tau$. Both covariances have the same values for $\tau=0,0.5$ and $1 \mathrm{px}$, leading to no interpolation bias at these points, while their maximum differences are near 0.2 and $0.8 \mathrm{px}$. To minimize the number of images required to build the covariance terms, we introduced a quartic model to represent the evolution of the term $\operatorname{cov}\left(e_{i}(\tau), T\left(\beta_{i}\right)\right)$. The other term $\operatorname{cov}\left(e_{i}(\tau), T\left(\beta_{i+1}\right)\right)$ is determined using the symmetry property with respect $\tau=0.5 \mathrm{px}$. The fourth-order polynomial is fitted using three images corresponding to $\tau=0.24,0.5$ and $0.76 \mathrm{px}$, respectively, and imposing a null covariance for $\tau=0$ and 1 px. The predictions of this model are also plotted in Fig. 10 , and the points used for the model fitting are represented

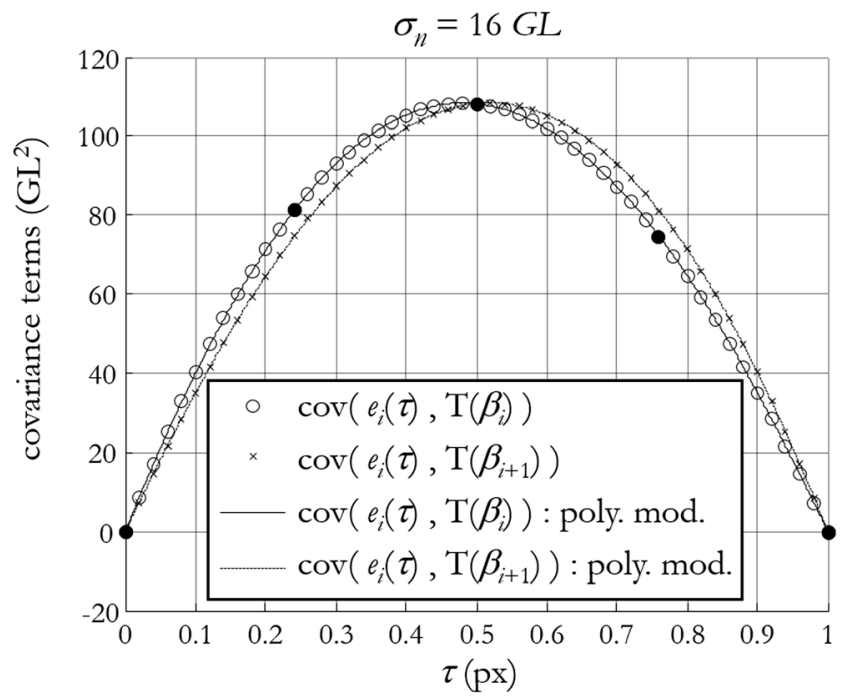

Fig. 10 Evolution of the covariance terms in equation (24). "•" denotes the data chosen to identify the polynomial model 
with filled circles. This simplistic (three-parameters) model is in very good agreement with the observation from simulated images.

Figure 11 presents the evolution of the expectation $\mathrm{E}[\Delta u]$ with the imposed displacement in the case of noiseless images $\left(\sigma_{n}=0 \mathrm{GL}\right.$ in equation (24) so that $\mathrm{E}_{\text {noise }}=0$ ). By substituting the expression of the covariance terms associated with the quartic model in equation (24), the systematic error evolution $\mathrm{E}[\Delta u]$ can be computed for any imposed displacement using a set of only three images. The systematic error obtained in this way is very close to the numerical results.

Due to the covariance evolution presented in Fig. 10 and because $\sigma_{n}=0$, a quasi-sinusoidal S-shaped curve is obtained, corresponding solely to the interpolation bias error $\mathrm{E}_{\text {interp. }}$ This curve presents a maximum amplitude for $\tau$ near 0.2 and $0.8 \mathrm{px}$ and a value equal to zero for $\tau$ equal to $0,0.5$ and $1 \mathrm{px}$. It stands to reason that the S-shape obtained from equation (24) depends on the image characteristics and on the assumptions made for the correlation criteria (here SSD) and for the image interpolation (here bi-linear). This result is then compared to the systematic error computed directly by DIC (see Fig. 11). One can clearly observe that both these quantities are very similar. This result shows that the proposed model described in equation (24) is consistent with the numerical results. In this case, the classical systematic error evolution can be directly predicted from sub-pixel shifted images without any DIC computation. It is also possible to propose and identify a simple (e.g., polynomial) model to estimate the dependence of the terms $\operatorname{cov}\left(e_{i}(\tau), T\left(X_{i}\right)\right)$

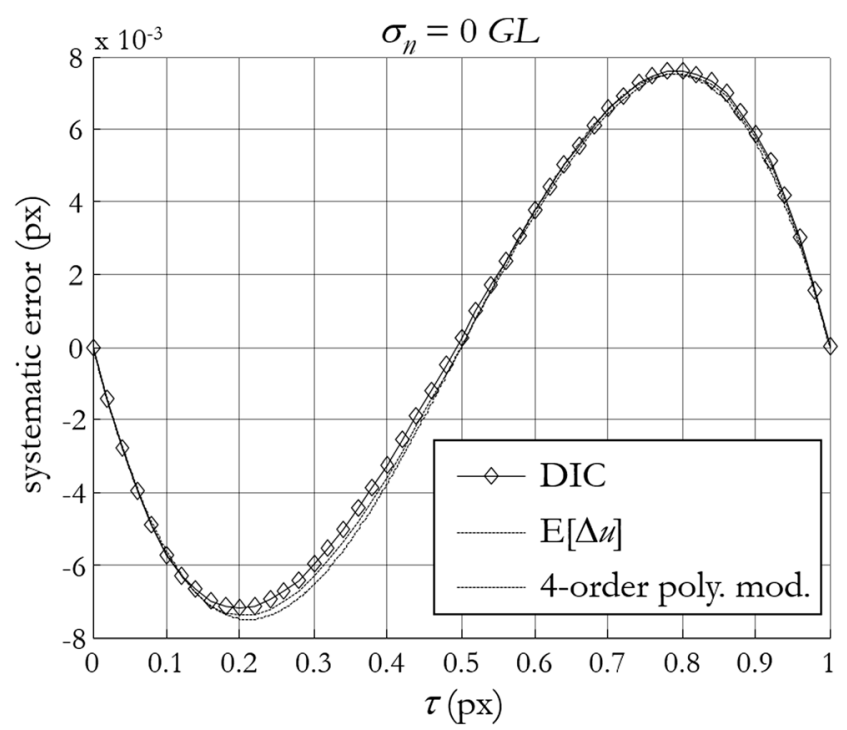

Fig. 11 Evolution of the systematic error with $\tau$ without image noise $\left(\sigma_{n}=0 \mathrm{GL}\right.$ and $\left.\mathrm{d}=16 \mathrm{px}\right)$ computed with DIC and given by equation (24) and $\operatorname{cov}\left(e_{i}(\tau), T\left(X_{i+1}\right)\right)$ with respect to $\tau$ to predict the shape of the systematic error using a reduced number of shifted images, without any DIC computation. The simplest approach consists of modelling the covariance terms illustrated in Fig. 10 by quartic polynomials. As the covariances are symmetric with respect to the axis $\tau=0.5$ and are null for $\tau=0 \mathrm{px}$ and $\tau=1 \mathrm{px}$, only three images for three imposed displacements (e.g., $\tau=0.24,0.5$ and $0.76 \mathrm{px})$ are necessary to identify this model.

The case of noisy images is presented in Fig. 12 for several values of $\sigma_{n}(2,4,8$ and 16 grey levels). Expectations $\mathrm{E}[\Delta u]$ are obtained from equation (24). They are compared to the result of systematic errors directly computed by DIC from the speckle images. The results for noiseless images are also superimposed in dashed lines: they represent the interpolation bias error $\mathrm{E}_{\text {interp. }}$ In the same manner, the dotted-dashed line represents the $\mathrm{E}_{\text {noise }}$ contribution.

The proposed model can predict the dependence of the systematic error on image noise except near the integer values of imposed displacement (i.e., where $\delta_{x}^{m}$ $\leq \tau \leq 1-\delta_{x}^{m}$ ) and for high noise levels. As discussed in section 3, when $\tau \leq \delta_{x}^{m}$, DIC results for noisy images are corrupted by the non-convexity of the SSD criterion. This situation is described in detail in the next section.

Following equation (24), the systematic error is the difference between the interpolation bias contribution $\mathrm{E}_{\text {interp }}$ (which is constant whatever the noise level) and the noise bias contribution $\mathrm{E}_{\text {noise }}$ (which is affined with $\tau$ and depends linearly on the noise variance $\sigma_{n}^{2}$ ). For relatively small noise levels (e.g., $\sigma_{n} \leq 2 \mathrm{GL}$ ) and $\delta_{x}^{m} \leq \tau \leq 1-\delta_{x}^{m}$, the amplitude of $\mathrm{E}_{\text {noise }}$ is similar to that of $E_{\text {interp. }}$ Consequently, the amplitude of the curve of $\mathrm{E}[\Delta u]$ presented in Fig. 12(a) is smaller than the one for the noiseless images. In this case, counterintuitively, adding image noise diminishes the bias amplitude. When the noise increases, the noise bias contribution becomes preponderant (see Fig. 12(c, d)). In each case, the slope of $\mathrm{E}_{\text {noise }}$ is equal to $-2 \sigma_{n}^{2} / \overline{\nabla T^{2}}$. For very high noise levels $\left(\sigma_{n}=16 \mathrm{GL}\right)$, a significant discrepancy is observed between both the simulated and the modelled systematic errors. This discrepancy is interpreted as a lack of statistic representativeness for small subsets with a high noise level.

\section{Phenomenological Extension of the Model}

Using the same framework as the one detailed in section 3.4 (a bimodal population associated with two separated solutions close to integer values, i.e., $\tau \leq \delta_{x}^{m}$ 
), the systematic error is given by the expectancy of the error in displacement given by equation (25):

$$
\begin{aligned}
\overline{\Delta u}_{p}(\tau) & =E\left[u_{p}(\tau)\right]-\tau \\
& =\frac{1}{N_{0}}\left[m_{l}(\tau) N_{l}(\tau)+m_{r}(\tau) N_{r}(\tau)\right]-\tau
\end{aligned}
$$

From different formulae $(10,11,13)$ and using the chosen model for $\delta N(\tau)$, the expectation can be calculated for the linear model (equation (26)) and for the exponential model (equation (27)):

$\overline{\Delta u}_{p}(\tau)=\frac{\tau}{2}+\frac{1}{2 \delta_{X}^{t h}} \tau^{2}$

$\overline{\Delta u}_{p}(\tau)=-\frac{\tau}{2}+\frac{a}{N_{0}}\left(1-e^{-b \tau}\right)\left(\tau+2 \delta_{X}^{t h}\right)$

Both expressions are consistent with curves from simulated images (see Fig. 13): they increase and are null for $\tau=0$ px. The discrepancy between the model and numerical results increases with $\tau$. For small values of $\tau$, the model is correct because the loss of convexity of correlation criterion is well considered. For higher values of $\tau$ $\left(\delta_{x}^{m} \leq \tau \leq 1-\delta_{x}^{m}\right)$, the previous model (equation (24)) correctly predicts the systematic error. For intermediate values of $\tau$, these two effects contribute to the error with a relative weight depending on $\tau$.

In conclusion, the two effects presented in this section and the previous section can be gathered to propose a predictive model for the systematic error expressed as the minimum of equations (24) and (25) for an imposed displacement lying between 0 and $0.5 \mathrm{px}$ (the central symmetry with respect to $\tau=0.5 \mathrm{px}$ gives the expression for $0.5<\tau<1$ ):

$$
\overline{\Delta u}(\tau)=\min \left(\mathrm{E}[\Delta u(\tau)], \overline{\Delta u}_{p}(\tau)\right)
$$

It is then possible to identify the model with a limited number of images: the initial image $(\tau=0)$ to get grey level gradients, and three images (for three imposed displacements) to determine the variation of $E_{\text {interp }}$ with respect to $\tau$.

Note that for high-noise levels, a simpler model can be proposed because the interpolation error term $E_{\text {interp }}$ becomes negligible:
Fig. 12 Evolution of the systematic error with $\tau$ for various image noise levels computed with DIC and given by equation (24) (a)

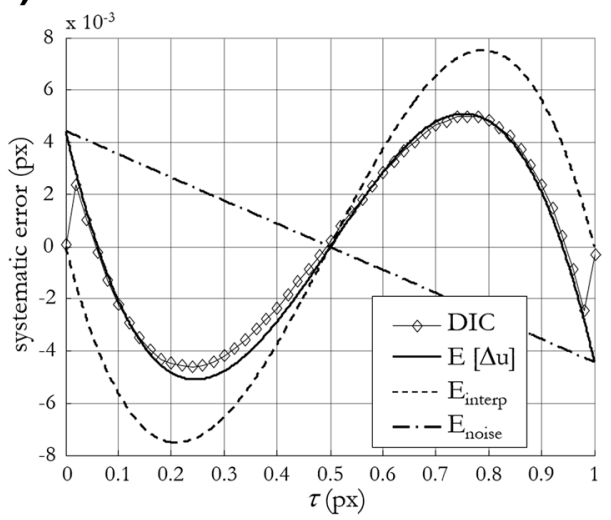

(c)

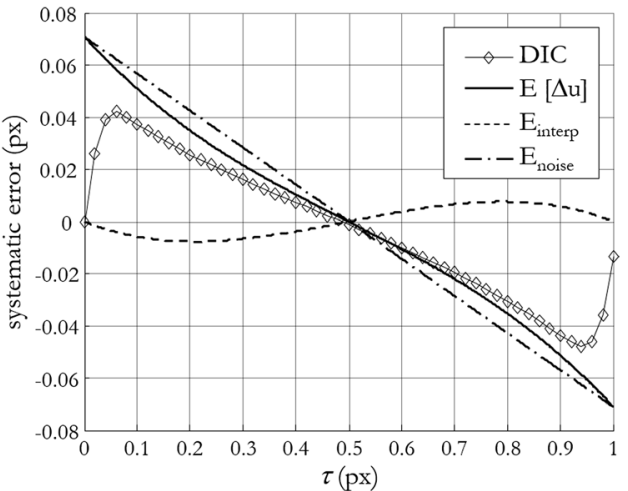

(b)

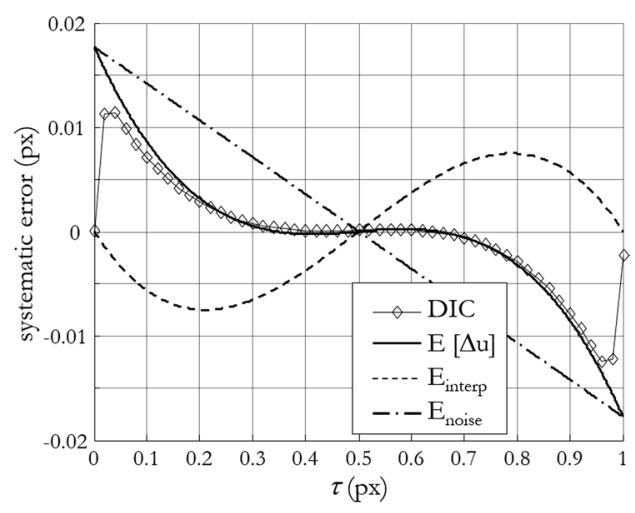

(d)

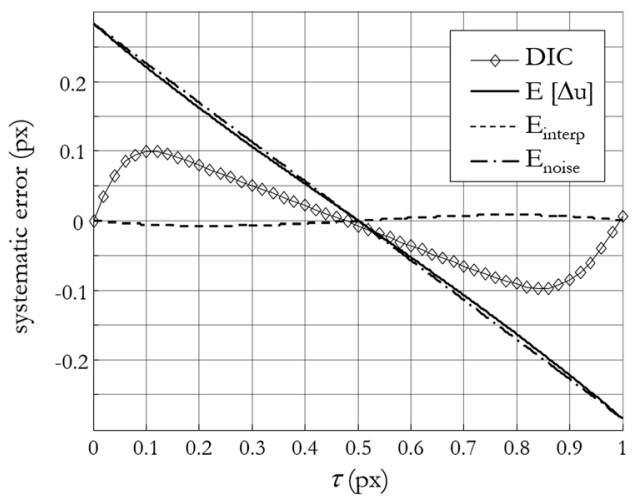




$$
\left.\left.\overline{\Delta u}(\tau)=\min \frac{\sigma_{n}^{2}(1-2 \tau)}{\overline{\nabla T^{2}}}, \frac{\tau}{2}+\frac{2 \sigma_{n}^{2}+\overline{\nabla T^{2}}}{2 \sigma_{n}^{2}} \tau^{2} \text { or }-\frac{\tau}{2}+\frac{a}{N_{0}}\left(1-e^{-b \tau}\right) \tau+\frac{2 \sigma_{n}^{2}}{2 \sigma_{n}^{2}+\overline{\nabla T^{2}}}\right)\right)
$$

In that case, for a known noise level, a single image can be necessary to identify the model parameters.

\section{Conclusions}

In this paper, new prediction models of DIC errors are proposed for the ultimate error regime, when the shape function is rich enough to represent the actual displacement field. The usual case of pure sub-pixel translations is studied using the same methodology as the one proposed in [21] from synthetic speckle-pattern 8-bit images. The statistical analysis of differences between imposed and measured displacements gives the evolutions of systematic and random errors, which are compared to existing models from the literature. Two models have been proposed in the literature: the first one predicts the random error according to image noise, the subset size and the

(a)

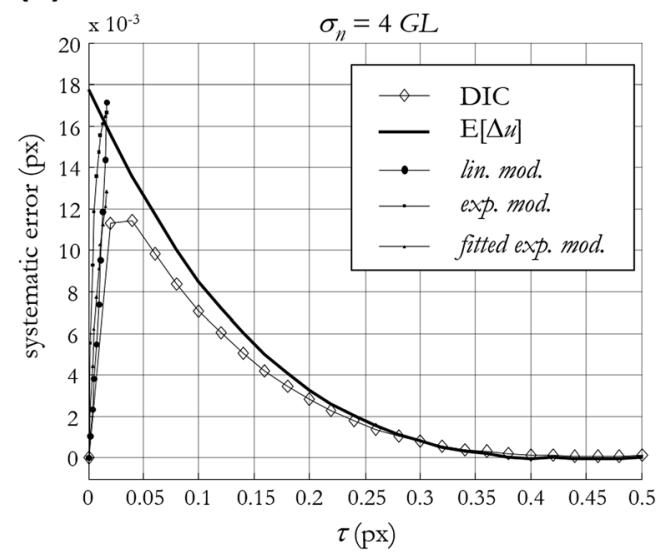

(b)

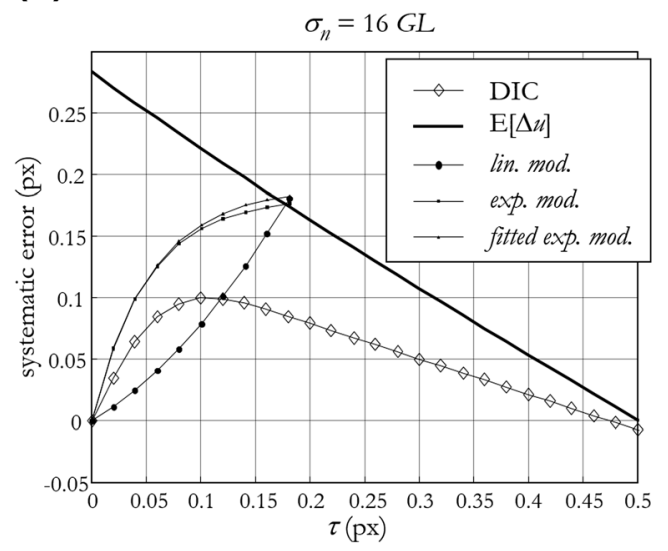

Fig. 13 Evolution of the systematic error and models with $\tau$ for two image noise levels grey level gradients; the second one adds an expression of systematic error mainly depending on fractional displacement and noise level, as observed for simulated data. Nevertheless, we note differences between the predictions of these models and numerical results obtained from synthetic images: random error is independent of fractional displacement and systematic error does not decrease for values close to integer displacements whatever the noise level.

These trends are not considered by classical models. They are linked to loss of the convexity of correlation criterion for noisy images: two pseudo-Gaussian distributions of measured displacements appear with similar standard deviations, different means and distribution frequencies that change according to imposed fractional displacement. The evolutions of the latter parameters give directly the evolutions of both systematic and random errors. We proposed a statistical description of these two evolutions that complements both models (systematic and random errors) and wrote two closed form expressions for the systematic and random errors for a linear interpolation scheme depending only on image properties and imposed displacement. A consistent description of errors was then obtained whatever the imposed displacement and noise level. The two models developed here are built with a very limited number of parameters that can be identified from a very small number of images (one to four images to accurately predict the evolutions of systematic and random errors).

Furthermore, it is important to emphasize that the proposed models are built with an approach similar to the one usually used to determine systematic and random errors from experimental data: subpixel translation displacements are imposed to a given image, the subset size is small and the statistic variability of situations arises from an analysis of the displacement of several subsets in an image.

To conclude, this study allows obtaining an enriched model for the both types of DIC errors. The random error well models the DIC errors obtained from simulated images even for a high level of noise. The model of bias error allows us to well describe the behaviour of DIC close to integer values of imposed displacement. Nevertheless, this model is less realistic for high values of noise, but such levels are unusual in practice. Finally, this work provides a response to the assessment of errors for the ultimate error regime and for a "perfect" measurement chain, its sole imperfection being related to image noise and interpolation bias. Naturally, deviations from 
this ideal situation may generate other contributions that could be significantly higher than the ones presented here.

Acknowledgements The authors gratefully acknowledge the French CNRS (National Centre for Scientific Research) for supporting this research through the GDR2519 research network "Mesures de Champs et Identification en Mécanique des Solides".

\section{References}

1. Sutton MA, Orteu J-J, Schreier HW (2009) Image correlation for shape, motion and deformation measurements - basic concepts, theory and applications. Springer, New York

2. Pan B, Qian K, Xie H, Asundi A (2009) Two-dimensional digital image correlation for in-plane displacement and strain measurement: a review. Meas Sci Technol 20:062001

3. Grédiac M, Hild F (eds) (2012) Full-field measurements and identification in solid mechanics. Wiley-ISTE, London

4. Orteu JJ (2009) 3-D computer vision in experimental mechanics. Opt Lasers Eng 47(3):282-291

5. Sutton MA, Yan JH, Tiwari V, Schreier HW, Orteu JJ (2008) The effect of out-of-plane motion on 2D and 3D digital image correlation measurements. Opt Lasers Eng 46:746-757

6. Germaneau A, Doumalin P, Dupré JC (2007) 3D strain field measurement by correlation of volume images using scatered light: recording of images and choice of marks. Strain 43:207-218

7. Forsberg F, Mooser R, Arnold M, Hack E, Wyss P (2008) 3D micro-scale deformations of wood in bending: synchrotron radiation $\mu \mathrm{CT}$ data analyzed with digital volume correlation. J Struct Biol 164(3):255-262

8. Tran H, Doumalin P, Delisee C, Dupre JC, Malvestio J, Germaneau A (2013) 3D mechanical analysis of low-density wood-based fiberboards by X-ray microcomputed tomography and digital volume correlation. J Mater Sci 48(8):3198-3212

9. Lenoir N, Bornert M, Desrues J, Bésuelle P, Viggiani G (2007) Volumetric digital image correlation applied to X-ray microtomography images from triaxial compression tests on argillaceous rock. Strain 43(3):193-205

10. Notbohm J, Kim JH, Franck C, Maskarinec S, Tirrell D, Asthagiri A, Ravichandran G (2012) Three-dimensional traction force microscopy for studying cellular interactions with biomaterials. Procedia IUTAM 4:144-150

11. Zhong X, Spottiswoode BS, Meyer CH, Kramer CM, Epstein FH (2010) Imaging three-dimensional myocardial mechanics using navigator-gated volumetric spiral cine DENSE MRI. Magn Reson Med 64(4):1089-1097

12. Cheng P, Sutton M, Schreier H, McNeill S (2002) Full-field speckle pattern image correlation with B-spline deformation function. Exp Mech 42(3):344-352

13. Besnard G, Hild F, Roux S (2006) "Finite-element" displacement fields analysis from digital images: application to Portevin-Le Châtelier bands. Exp Mech 46(6):789-803

14. Hild F, Roux S (2006) Measuring stress intensity factors with a camera: integrated digital image correlation (I-DIC). CRAS 334(1):8-12

15. Haddadi H, Belhabib S (2008) Use of a rigid-body motion for the investigation and estimation of the measurement errors related to digital image correlation technique. Opt Lasers Eng 46:185-196

16. Chu TC, Ranson WF, Sutton MA, Peters WH (1985) Applications of digital image-correlation techniques to experimental mechanics. Exp Mech 25:232-244
17. Sutton MA, McNeill SR, Jang J, Babai M (1988) Effects of subpixel image restoration on digital correlation error estimates. Opt Eng 27:870-877

18. Choi S, Shah SP (1997) Measurement of deformations on concrete subjected to compression using Image correlation. Exp Mech 37: 307-313

19. Roux S, Hild F (2006) Stress intensity factor measurements from digital image correlation: post-processing and integrated approaches. Int J Fract 140:141-157

20. Wang YQ, Sutton MA, Bruck HA, Schreier HW (2009) Quantitative error assessment in pattern matching: effects of intensity pattern noise, interpolation, strain and image contrast on motion measurements. Strain 45:160-178

21. Bornert M, Brémand F, Doumalin P, Dupré J-C, Fazzini M, Grédiac M, Hild F, Mistou S, Molimard J, Orteu J-J, Robert L, Surrel Y, Vacher P, Wattrisse B (2009) Assessment of digital image correlation measurement errors: methodology and results. Exp Mech 49: 353-370

22. Pan B, Wang B (2016) Digital image correlation with enhanced accuracy and efficiency: a comparison of two subpixel registration algorithms. Exp Mech 56:1395-1409

23. Wang Y, Lava P, Reu P, Debruyne D (2016) Theoretical analysis on the measurement errors of local 2D DIC: part I temporal and spatial uncertainty quantification of displacement measurements. Strain 52:110-128

24. Doumalin P, Bornert M, Caldemaison D (1999) Microextensometry by image correlation applied to micromechanical studies using the scanning electron microscopy. Proc. International conference on advanced technology in experimental mechanics, Ube City, Japan, pp 81-86

25. Wattrisse B, Chrysochoos A, Muracciole J-M, Némoz-Gaillard M (2000) Analysis of strain localization during tensile tests by digital image correlation. Exp Mech 41:29-39

26. Schreier HW, Braasch JR, Sutton MA (2000) Systematic errors in digital image correlation caused by intensity interpolation. Opt Eng 39:2915-2921

27. Zhou P, Goodson KE (2001) Subpixel displacement and deformation gradient measurement using digital image/speckle correlation (DISC). Opt Eng 40:1613

28. Wang Y, Cuitino AM (2002) Full-field measurements of heterogeneous deformation patterns on polymeric foams using digital image correlation. Int J Solids Struct 39:3777-3796

29. Schreier HW, Sutton MA (2002) Systematic errors in digital image correlation due to undermatched subset shape function. Exp Mech 42:303-310

30. Pan B, Xie H-M, Xu BQ, Dai FL (2006) Performance of sub-pixel registration algorithms in digital image correlation. Meas Sci Technol 17:1615-1621

31. Lecompte D, Smits A, Bossuyt S, Sol H, Vantomme J, Van Hemelrijck D, Habraken AM (2006) Quality assessment of speckle patterns for digital image correlation. Opt Lasers Eng 44:11321145

32. Triconnet K, Derrien K, Hild F, Baptiste D (2009) Parameter choice for optimized digital image correlation. Opt Lasers Eng 47:728-737

33. Hua T, Xie H, Wang S, Hu Z, Chen P, Zhang Q (2011) Evaluation of the quality of a speckle pattern in the digital image correlation method by mean subset fluctuation. Opt Laser Technol 43:9-13

34. Pan B, Lu Z, Xie H (2010) Mean intensity gradient: an effective global parameter for quality assessment of the speckle patterns used in digital image correlation. Opt Lasers Eng 48:469-477

35. Sousa AMR, Xavier J, Vaz M, Morais JJL, Filipe VMJ (2011) Cross-correlation and differential technique combination to determine displacement fields. Strain 47:87-98

36. Bornert M, Doumalin P, Dupré J-C, Poilâne C, Robert L, Toussaint E, Wattrisse B (2012) Short remarks about synthetic image generation in the context of the assessment of sub-pixel accuracy of 
digital image correlation. Proc. International Conference on Experimental Mechanics (ICEM'15), Porto, Portugal

37. Reu P (2011) Experimental and numerical methods for exact subpixel shifting. Exp Mech 51:443-452

38. Zhang J, Cai Y, Ye W, Yu TX (2011) On the use of the digital image correlation method for heterogeneous deformation measurement of porous solids. Opt Lasers Eng 49(2):200-209
39. Amiot F, Bornert M, Doumalin P, Dupré J-C, Fazzini M, Orteu J-J, Poilâne C, Robert L, Rotinat R, Toussaint E, Wattrisse B, Wienin JS (2013) Assessment of digital image correlation measurement accuracy in the ultimate error regime: main results of a collaborative benchmark. Strain 49(6):483-496 